\title{
Influence of anisotropy on heterogeneous nucleation
}

\author{
A. Mariaux*, M. Rappaz \\ Laboratoire de Simulation des Matériaux, École Polytechnique Fédérale de Lausanne, EPFL-STI-IMX-LSMX, Station 12, CH-1015 Lausanne, Switzerland
}

Received 1 August 2010; accepted 7 October 2010

Available online 18 November 2010

\begin{abstract}
Heterogeneous nucleation is governed by the interplay of interfacial energies between a substrate, a solid and a liquid. Although the intensity of these energies can strongly change with the orientation of the nucleus for anisotropic media, this parameter is not taken into account in the available nucleation theories. In this paper, the Gibbs free energy barrier for nucleation is computed for an arbitrary solidliquid interface energy. It is shown that anisotropy favors particular orientations of the nucleus on the substrate. Experimental evidence from the zinc-aluminum system is given as an application of this extended nucleation theory. It also sheds new light on the texture of galvanized steel sheets.
\end{abstract}

(C) 2010 Acta Materialia Inc. Published by Elsevier Ltd. All rights reserved.

Keywords: Heterogeneous nucleation of phases; Interface energy; Anisotropy; Texture; Hot-dip galvanization

\section{Introduction}

In the early stages of solidification, the stability of a solid cluster of atoms in a melt is determined by the difference between two opposing contributions to the free energy of the system: (i) the decrease in volume energy resulting from the phase transition and (ii) the interfacial energy between the involved phases. Based on this analysis, several theories of nucleation have been developed in the past decades [1-3]. In all of these, the interfacial energy is assumed to be isotropic and thus the nuclei to be spheres, or caps thereof. Under these conditions, the orientation of a heterogeneous nucleus on a foreign substrate is irrelevant. However, as already mentioned by Taylor and Cahn [4], this parameter cannot be neglected if the anisotropy of the solid-liquid and/or solid-substrate interfacial energies becomes large. It can even have such a large effect that nucleation is favored for some orientations and hindered for others. This is shown from a general point of view in this paper by extending the classical model of heteroge-

\footnotetext{
* Corresponding author. Tel.: +41 21693 2938; fax: +41 216935890.

E-mail addresses: aurele.mariaux@epfl.ch (A. Mariaux), michel.rappaz @epfl.ch (M. Rappaz).
}

neous nucleation to account for the anisotropy of the solid-liquid interfacial energy. Under its influence, the equilibrium shape of a crystal fully surrounded by liquid deviates from that of a sphere. Thus, even if the substrate is isotropic, the shape of the heterogeneous nucleus is no longer a spherical cap, and the nucleation energy barrier becomes a function of its orientation with respect to the substrate. In the first part, the orientation dependence of the nucleation energy is computed based on the $\vec{\xi}$-vector formalism of Hoffman and Cahn [5]. In the second part, applications of this theory to hot-dip galvanized $\mathrm{Zn}-\mathrm{Al}$ coatings are presented. Zinc has a high anisotropy and the coatings exhibit a preferential basal orientation $\left(\begin{array}{llll}0 & 0 & 0 & 1\end{array}\right\}$ plane parallel to the surface of the nucleation substrate). Several observations on this system clearly illustrate the influence of the solid-liquid interfacial energy anisotropy on heterogeneous nucleation.

\section{Computation of the energy of a nucleus}

\subsection{Nucleus geometry}

Let us consider a cluster of atoms with a solid structure, sitting on a planar, and non-reacting, foreign substrate. 
Due to the very small size of the cluster, chemical potential gradients along the solid-liquid interface are leveled out very quickly by diffusion and the solid is assumed to take its equilibrium shape. The corresponding geometry can be computed from the solid-liquid interfacial energy, $\gamma_{s l}\left(\vec{n}_{s l}\right)$, with the help of the $\vec{\xi}$-vector formalism [5,6]. Given $\gamma_{s l}\left(\vec{n}_{s l}\right)=\gamma_{s l}^{\circ} \eta\left(\vec{n}_{s l}\right)$, with $\eta\left(\vec{n}_{s l}\right)$ the anisotropy of $\gamma_{s l}$ and $\vec{n}_{s l}$ the unit vector normal to the solid-liquid interface, the equilibrium shape of a solid particle surrounded only by its melt is homothetic to the parametric surface:

$\vec{\xi}_{s l}\left(\vec{n}_{s l}\right)=\nabla\left(r \gamma_{s l}\left(\vec{n}_{s l}\right)\right)$

where $\vec{r}$ is the extension of $\vec{n}_{s l}$ to $\mathbb{R}^{3}$ with $r=|\vec{r}|$ and $\vec{r}=r \vec{n}_{s l}$.

If the particle is located on a foreign medium, this shape is truncated by a plane parallel to the substrate, at a distance $\Delta \gamma=\gamma_{l f}-\gamma_{f s}$ from its center (see Fig. 1), where the indices $f, s$ and $l$ indicate the foreign substrate, the solid and the liquid phase, respectively (i.e. $\gamma_{l f}$ and $\gamma_{f s}$ are the interfacial energies between liquid and substrate, and between substrate and solid, respectively). The quantity $\Delta \gamma$ can be either isotropic or anisotropic, depending on the properties of $\gamma_{l f}$ and $\gamma_{f s}$. In particular, even if the substrate is isotropic, $\gamma_{f s}$ and thus $\Delta \gamma$ can change with the orientation of the solid-substrate interface due to the anisotropic solid alone. In this study, $\Delta \gamma$ will be assumed to be isotropic, so that it can be varied as a free parameter, independently of the orientation. However, all results can be easily adapted to the case of anisotropic $\Delta \gamma$ by setting $\Delta \gamma=\Delta \gamma\left(\vec{n}_{f s}\right)$ in the equations, with $\vec{n}_{f s}$ the unit vector normal to the solid-substrate interface.

In the $\vec{\xi}$-vector formalism, the wetting condition is expressed as [6]:

$\vec{\xi}_{s l}\left(\vec{n}_{s l}\right) \cdot \vec{n}_{f s}=\Delta \gamma$

where the indices $f, s$ and $l$ of the vectors $\vec{n}$ have the same meaning as above, but additionally indicate their direction: $\vec{n}_{i j}$ is a unit vector normal to the interface between media $i$ and $j$, which points out of $i$ into $j$.

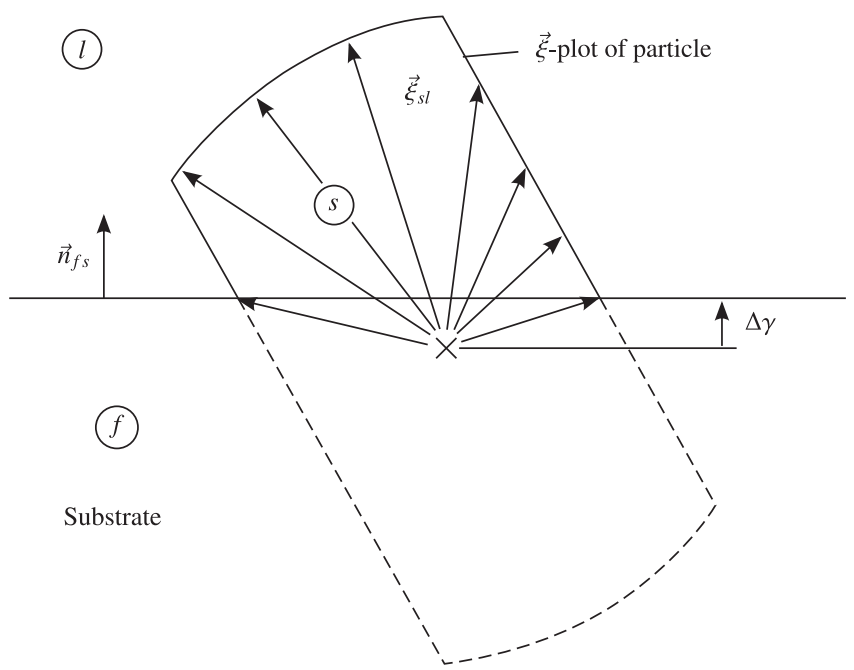

Fig. 1. Anisotropic particle on a flat surface (redrawn after Ref. [6]).
The whole bounding surface of the nucleus is made of the solid-liquid and the solid-substrate interfaces. Accordingly, we define its position, $\vec{X}$, as a combination of two functions. On the solid-liquid interface, $\vec{X}$ is given by

$\vec{X}\left(\vec{n}_{s l}\right)=\frac{X_{0}}{\gamma_{s l}^{\circ}} \vec{\xi}_{s l}\left(\vec{n}_{s l}\right)$

with $X_{0}$ a scaling factor describing the size of the nucleus. Along the solid-substrate interface, it is enough for the present study to note that $\vec{X}$ satisfies Eq. (2):

$\vec{X} \cdot \vec{n}_{f s}=\frac{X_{0}}{\gamma_{s l}^{\circ}} \Delta \gamma$

to comply with the wetting condition.

\subsection{Nucleus energy}

The Gibbs free energy of a nucleus, $\Delta G^{\text {het }}$, is given by the following integral:

$\Delta G^{\text {het }}=\rho \Delta g_{\mathrm{f}} V_{s}+\int_{A_{s l}} \gamma_{s l}\left(\vec{n}_{s l}\right) \mathrm{d} A-\Delta \gamma \int_{A_{f s}} \mathrm{~d} A$

where $\rho$ is the density, $\Delta g_{\mathrm{f}}$ is the specific Gibbs free energy difference between the solid and the liquid, $V_{s}$ is the volume of the nucleus, $A_{s l}$ is the solid-liquid interface area and $A_{f s}$ is the solid-substrate interface area. At small undercooling, the linear approximation $\Delta g_{\mathrm{f}}=-\Delta s_{\mathrm{f}} \Delta T$ can be used to compute the Gibbs free energy of fusion, where $\Delta s_{\mathrm{f}}$ is the specific entropy of fusion and $\Delta T$ the undercooling.

Although the geometry is much more complex in the present case, several properties of the isotropic nucleation model can be found again, which are shown below.

For a given nucleus size $X_{0}$, the volumes of the heterogeneous and homogeneous nuclei are proportional and their ratio depends only on the wetting conditions (given by $\Delta \gamma /$ $\gamma_{s l}^{\circ}$ ) and the orientation $\vec{n}_{f s}$ of the substrate, since these two parameters completely define how the equilibrium shape is truncated (Fig. 1). Thus, we have:

$\frac{V_{s}^{\text {het }}\left(X_{0}, \Delta \gamma / \gamma_{s l}^{\circ}, \vec{n}_{f s}\right)}{V_{s}^{\text {hom }}\left(X_{0}\right)}=f\left(\Delta \gamma / \gamma_{s l}^{\circ}, \vec{n}_{f s}\right), \quad \forall X_{0}$

where $\vec{n}_{f s}$ is the orientation of the substrate surface measured in the reference system of the nucleus crystallographic structure. In the isotropic theory of heterogeneous nucleation, the equivalent function $f(\theta)$, where $\theta$ is the wetting angle, is found through simple geometric considerations, but the generalized function $f\left(\Delta \gamma / \gamma_{s l}^{\circ}, \vec{n}_{f s}\right)$ cannot be computed explicitly, due to the much more complicated shape of the nucleus. It is nevertheless possible to extend a further property of isotropic nuclei to the present case, namely the fact that the Gibbs free energies of the heterogeneous and homogeneous nuclei are in the same ratio as their volumes:

$\frac{\Delta G^{\text {het }}}{\Delta G^{\text {hom }}}=\frac{V_{s}^{\text {het }}}{V_{s}^{\text {hom }}}=f\left(\Delta \gamma / \gamma_{s l}^{\circ}, \vec{n}_{f s}\right)$ 
We demonstrate this by using first Eq. (1), from which can be deduced that:

$\gamma_{s l}\left(\vec{n}_{s l}\right)=\vec{\xi}_{s l}\left(\vec{n}_{s l}\right) \cdot \vec{n}_{s l}$

With this result and Eq. (4), the last two terms in Eq. (5) transform into:

$\int_{A_{s l}} \frac{\gamma_{s l}^{\circ}}{X_{0}} \vec{X} \cdot \vec{n}_{s l} \mathrm{~d} A+\int_{A_{s f}} \frac{\gamma_{s l}^{\circ}}{X_{0}} \vec{X} \cdot \vec{n}_{s f} \mathrm{~d} A=\frac{\gamma_{s l}^{\circ}}{X_{0}} \int_{A_{s}} \vec{X} \cdot \vec{n}_{s} \mathrm{~d} A$

Please note that the negative sign in the last term of Eq. (5) has been integrated into the orientation of the normal to the substrate $\left(\vec{n}_{s f}=-\vec{n}_{f s}\right)$. The two integrals on the left-hand side of Eq. (9) transform into an integral over the whole surface $A_{s}$ of the nucleus, i.e. over the solidliquid and solid-substrate interfaces. The outward-pointing normal to this surface is simply labeled $\vec{n}_{s}$. Through the divergence theorem, the right-hand side of Eq. (9) is also equal to:

$\frac{\gamma_{s l}^{\circ}}{X_{0}} \int_{V_{s}} \operatorname{div} \vec{X} \mathrm{~d} V=\frac{3 \gamma_{s l}^{\circ}}{X_{0}} V_{s}$

Thus, the surface energy contribution to the Gibbs free energy of a nucleus scales with its volume. Substituting this expression back into Eq. (5) leads to:

$\Delta G^{\text {het } / \text { hom }}=\left(-\rho \Delta s_{\mathrm{f}} \Delta T+\frac{3 \gamma_{s l}^{\circ}}{X_{0}}\right) V_{s}^{\text {het } / \text { hom }}$

both for a heterogeneous and a homogeneous nucleus (the latter case is obtained by setting $A_{s l}=A_{s}$ and $A_{f s}=0$ in Eq. (5), which directly gives the right-hand side of Eq. (9)). A simple division yields Eq. (7).

The volume therefore scales with the third power of the nucleus size, $X_{0}$, and the surface terms with its square, as they do with a spherical cap. This can be made apparent in Eq. (11) by defining a dimensionless volume of the nucleus, $\widehat{V}_{s}^{\mathrm{het} / \mathrm{hom}}=V_{s}^{\mathrm{het} / \mathrm{hom}} / X_{0}^{3}$, which is independent of $X_{0}$, and substituting:

$\Delta G^{\text {het } / \text { hom }}=\left(-\rho \Delta s_{\mathrm{f}} \Delta T X_{0}^{3}+3 \gamma_{s l}^{\circ} X_{0}^{2}\right) \widehat{V}_{s}^{\text {het } / \text { hom }}$

This expression admits a maximum at:

$X_{0}=X_{0 c}=\frac{2 \gamma_{s l}^{\circ}}{\rho \Delta s_{\mathrm{f}} \Delta T}$

which generalizes the equation of the critical radius found for an isotropic nucleus. Introducing this into Eq. (12) gives the nucleation energy:

$$
\begin{aligned}
\Delta G_{c}^{\mathrm{het}} & =\Delta G_{c}^{\mathrm{hom}} f\left(\frac{\Delta \gamma}{\gamma_{s l}^{\circ}}, \vec{n}_{f s}\right) \\
& =\frac{4 \gamma_{s l}^{\circ}{ }^{3}}{\left(\rho \Delta s_{\mathrm{f}} \Delta T\right)^{2}} \widehat{V}_{s}^{\mathrm{hom}} f\left(\frac{\Delta \gamma}{\gamma_{s l}^{\circ}}, \vec{n}_{f s}\right)
\end{aligned}
$$

for a heterogeneous nucleus, where $\widehat{V}_{s}^{\text {hom }}=V_{s}^{\text {hom }} / X_{0}^{3}$ is the dimensionless volume of the homogeneous nucleus.
Eq. (14) is very similar to that obtained with an isotropic nucleus. The latter is found again by setting $X_{0}$ to the radius of the spherical cap and $\widehat{V}_{s}^{\text {hom }}=\frac{4}{3} \pi$. The parameter $\Delta \gamma / \gamma_{s l}^{\circ}$ in function $f$ is then proportional to the cosine of the wetting angle $\theta$ between the solid and the substrate. The main difference between Eq. (14) and former models is the introduction of the orientation dependence of $f$ through $\vec{n}_{f s}$.

Unlike a spherical cap, the part of the nucleus volume that remains after truncation does not depend only on the position of the cutting plane with respect to its center, but also on the direction of the cut. Therefore, $\widehat{V}_{s}^{\text {het }}$ and $f$ are dependent on the orientation of the nucleus on the substrate. This results in the nucleation energy (or critical volume of the nucleus) being lower for some orientations, thus favoring nucleation with these orientations.

\section{Application to zinc}

\subsection{Solid-liquid interfacial energy and its anisotropy}

The anisotropy of the solid-liquid interfacial energy of zinc (hexagonal close-packed structure) was determined from measurements of the quenched equilibrium shape of liquid droplets in a solid matrix [7]. These were not spheres, but lenses whose faces met at a sharp angle. No 6-fold symmetry was visible within the $\left.\begin{array}{lllll}0 & 0 & 0 & 1\end{array}\right\}$ plane. Accordingly, an expression containing only the first two orders of hexagonal harmonics was constructed:

$\gamma_{\mathrm{Zn}}(\vec{n})=\gamma_{s l}^{\circ}\left(1+a_{2}^{0} \mathscr{H}_{2}^{0}(\vec{n})+a_{4}^{0} \mathscr{H}_{4}^{0}(\vec{n})\right)$

where

$\mathscr{H}_{2}^{0}(\vec{n})=\frac{1}{4} \sqrt{\frac{5}{\pi}}\left(-1+3 n_{c}^{2}\right)$

$\mathscr{H}_{4}^{0}(\vec{n})=\frac{3}{16 \sqrt{\pi}}\left(3-30 n_{c}^{2}+35 n_{c}^{4}\right)$

with $n_{c}$ being the component of $\vec{n}$ parallel to the $\vec{c}$-axis of the zinc crystal. From the geometrical information in Ref. [7], values of $a_{2}^{0}=-1.026$ and $a_{4}^{0}=0.195$ were determined for the coefficients in Eq. (15). $\gamma_{s l}^{\circ}$ was computed from a measurement of the interfacial energy in $\left\{\begin{array}{llll}0 & 0 & 0 & 1\end{array}\right\}$ by Keşlioğlu and Maraşlı [8]. With the above values of $a_{2}^{0}$ and $a_{4}^{0}, \gamma_{s l}^{\{0001\}}=0.094 \mathrm{~J} \mathrm{~m}^{-2}$ leads to $\gamma_{s l}^{\circ}=0.182 \mathrm{~J} \mathrm{~m}^{-2}$. As these values are known, the shape of heterogeneous zinc nuclei on a substrate can be computed for any orientation and wetting condition. A few examples are given in Fig. 2.

\subsection{Nucleation energy}

The Gibbs free energy of a critical homogeneous nucleus was computed from Eqs. (14) and (15). Numerical integration provided a value of $\widehat{V}^{\text {hom }}=1.85$ for the dimensionless volume and, with $\rho \Delta s_{\mathrm{f}}=\rho L_{\mathrm{f}} / T_{m}=1.1 \times 10^{6} \mathrm{~J} \mathrm{~m}^{-3} \mathrm{~K}^{-1}$, where $L_{\mathrm{f}}$ is the specific enthalpy of fusion and $T_{m}$ the melting point: 


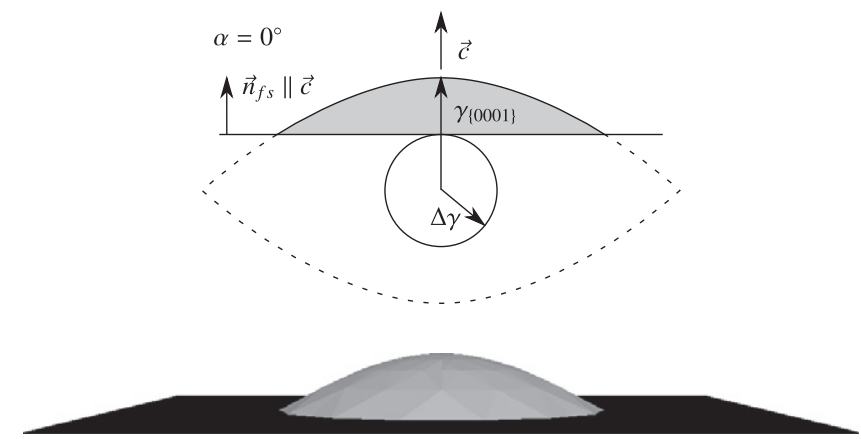

(a)

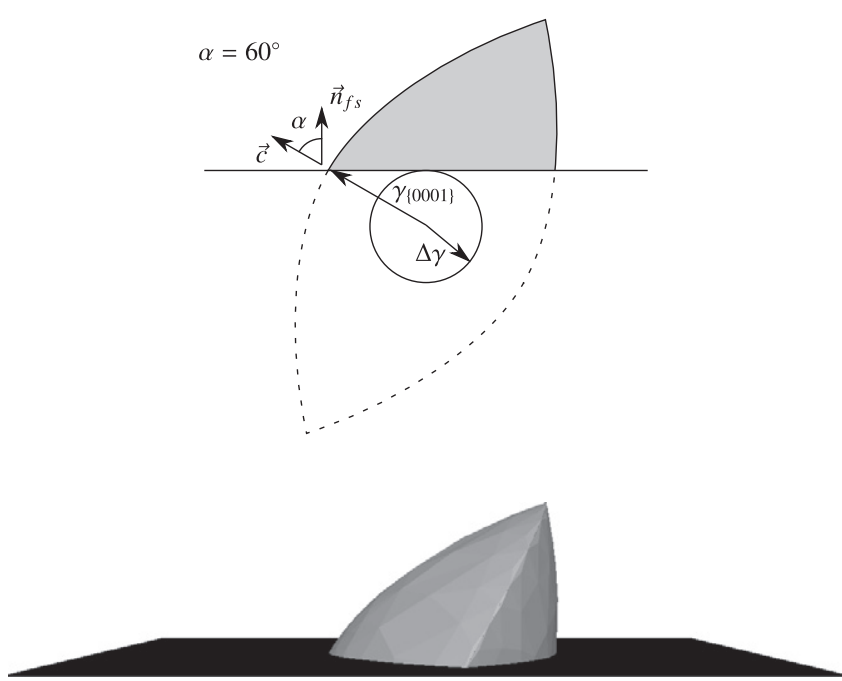

(b)

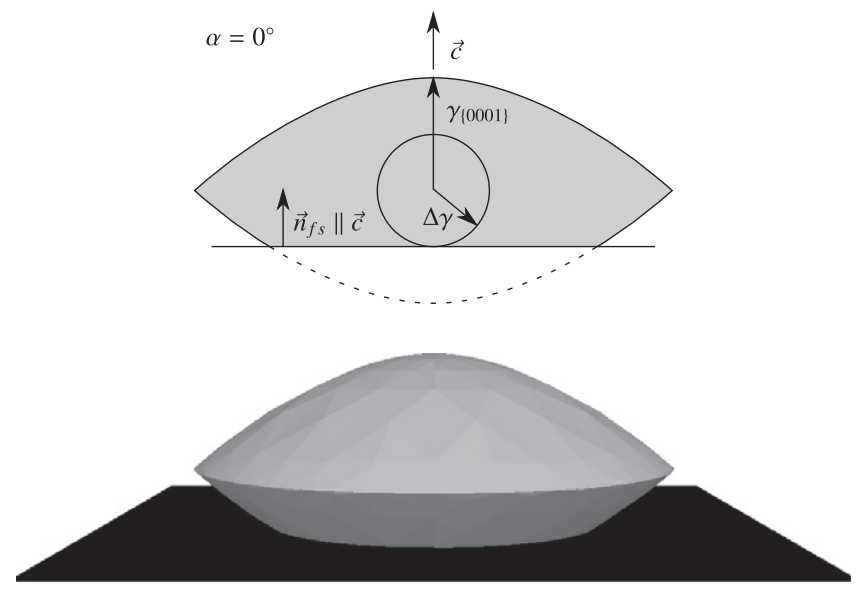

(c)
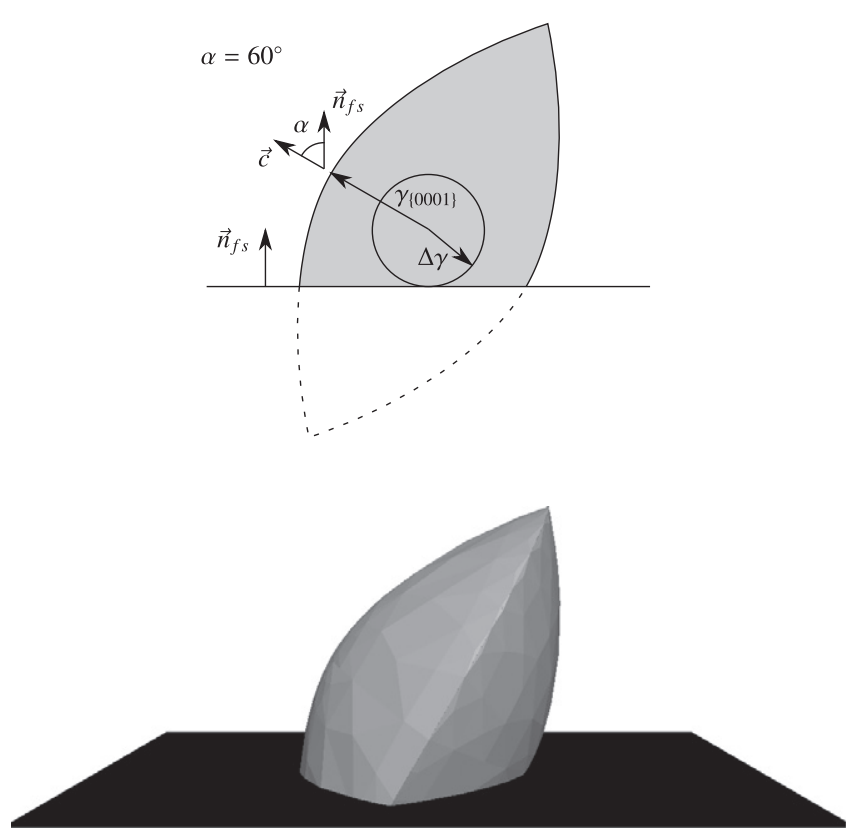

(d)

Fig. 2. Shape of zinc nuclei on a flat substrate for different orientations and wetting conditions: (a and b) $\Delta \gamma=0.5 \gamma_{s l}^{\circ}$ and (c and d) $\Delta \gamma=-0.5 \gamma_{s l}^{\circ}$.

$\Delta G_{c}^{\text {hom }}(\Delta T)=\frac{4 \times 10^{-14} \mathrm{~J} \mathrm{~K}^{2}}{(\Delta T)^{2}}$

The Gibbs free energy of a heterogeneous nucleus was seen above to depend on its orientation. In the case of zinc, since the equilibrium shape is invariant with a rotation about the $\vec{c}$-axis, only the angle $\alpha$ between $\vec{c}$ and the normal to the substrate plane, $\vec{n}_{f s}$, is relevant (see Fig. 2). The function $f\left(\Delta \gamma / \gamma_{s l}^{\circ}, \alpha\right)$ was computed by numerical integration of $\widehat{V}^{\text {het }}\left(\Delta \gamma / \gamma_{s l}^{\circ}, \alpha\right)$. The results are shown in Fig. 3 .

The value of $f$ strongly decreases when wetting of the solid on the substrate is improved (larger $\Delta \gamma$ ). In the extreme case $\Delta \gamma>\gamma_{s l}\left(\vec{n}_{f s}\right)$, i.e. perfect wetting, the nucleation energy goes to 0 . Since $\gamma_{s l}\left(\vec{n}_{f s}\right)+\gamma_{f s}<\gamma_{l f}$, a stable layer of solid spontaneously forms at the substrate-liquid interface to decrease its energy, either in orientation $\vec{n}_{f s}$ or in any other orientation $\vec{n}_{f s}^{\prime}$ such that $\gamma_{s l}\left(\vec{n}_{f_{s}}^{\prime}\right)<\gamma_{s l}\left(\vec{n}_{f s}\right)$. At a value $\Delta \gamma=0.5 \gamma_{s l}^{\circ}$, Fig. 2a and b show the volume of a heterogeneous nucleus when $\alpha=0$ (basal configuration) and $\alpha=60^{\circ}$, respectively. As can be seen, the volume of the nucleus, and thus its energy barrier is considerably reduced in the basal configuration. The situation $\Delta \gamma=-0.5 \gamma_{s l}^{\circ}$, i.e., not very favorable wetting condition of the solid on the foreign substrate, is shown in Fig. 2c and $\mathrm{d}$ for the same orientations. The situation is now reversed, as can be seen in the curves of Fig. 3: the volume and thus the energy barrier of the nucleus is reduced for the non-basal configuration. For neutral wetting, i.e. $\Delta \gamma=0$, the equilibrium shape crystal is cut in two equal parts and $f$ becomes independent of $\alpha$ (or $\vec{n}_{f s}$ ). Finally, the condition $\Delta \gamma<-\gamma_{s l}\left(\vec{n}_{f s}\right)$ prevents any wetting in orientation $\vec{n}_{f s}$ (and in any other orientation $\vec{n}_{f_{s}}^{\prime}$ such that 


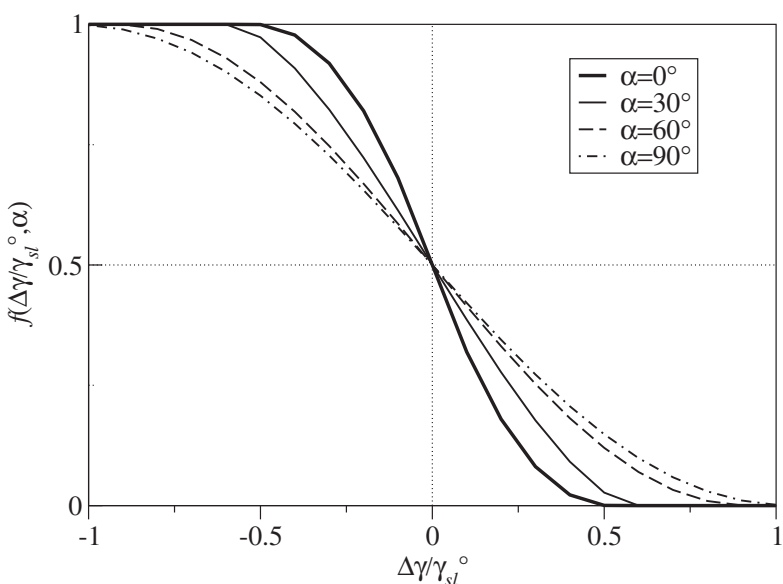

Fig. 3. Ratio of the Gibbs free energies associated with heterogeneous and homogeneous nuclei, as a function of the wetting conditions, for different orientations.

$\left.\gamma_{s l}\left(\vec{n}_{f s}^{\prime}\right)>\gamma_{s l}\left(\vec{n}_{f s}\right)\right)$ because the energy of the interface is lowered by adding a liquid film in between the solid and the substrate. When $\Delta \gamma<-\max _{\vec{n}_{f s}}\left(\gamma_{s l}\left(\vec{n}_{f s}\right)\right)$, this prevents any wetting on the substrate of a nucleus of arbitrary orientation. In all cases where wetting is prevented, since the nucleus is surrounded only by the liquid, it is homogeneous and $f\left(\Delta \gamma / \gamma_{s l}^{\circ}, \alpha\right)=1$.

The variation of $f$ with $\alpha$ is smaller than with $\Delta \gamma$, but it still has a significant influence. When the solid has a higher affinity to the substrate than the liquid $(\Delta \gamma>0)$, the energy needed to nucleate a grain with $\alpha=0^{\circ}$ is up to $50 \%$ smaller than that required for a grain with $\alpha=90^{\circ}$, for the same value of $\Delta \gamma / \gamma_{s l}^{\circ}$ (see Fig. 3). Thus, basal grains are strongly favored. Conversely, if the solid has less affinity to the substrate than the liquid $(\Delta \gamma<0)$, the reverse effect is expected: basal grains have a higher nucleation energy than those with other orientations.

This investigation of $f\left(\Delta \gamma / \gamma_{s l}^{\circ}, \alpha\right)$ shows that the anisotropy of $\gamma_{s l}$ can introduce a strong bias into the distribution of grain orientations. The direction and amount of this shift cannot be predicted from the properties of the solid-liquid interface alone, since they also depend on $\Delta \gamma$. They are therefore system properties and not intrinsic to the considered alloy. This becomes even more true if the substrate-solid interface energy is also anisotropic. In this case, $\Delta \gamma$ becomes dependent on $\alpha$ as well and the influence of orientation on the nucleation energy can be increased or damped as a consequence of this superposition.

\subsection{Experimental observations}

The effects of anisotropy on nucleation described in the previous section are very well observed on the free surface of a zinc melt. Through a combination of the anisotropies of $\gamma_{s l}$ and $\gamma_{f s}$, the formation of grains with their $\left\{\begin{array}{llll}0 & 0 & 0 & 1\end{array}\right\}$ plane parallel to the surface is strongly favored. This is illustrated here with the help of three examples, one for a sessile drop configuration and two for hot-dip galvanized coatings.

\subsubsection{Rose grain patterns}

In hot-dip galvanized coatings which were remelted in an infrared (IR) furnace [9], zinc grains were found, which had grown radially from a small round cavity (Fig. 4). They were called "rose patterns", since their morphology recalls the arrangement of petals in a flower. Interestingly, the central hole was delimited by facets, which were all perpendicular to the $\left\langle\begin{array}{llll}0 & 0 & 0 & 1\end{array}\right\rangle$ axis of the corresponding grain in a pole figure (see bottom of Fig. 4). The cavity was in fact entirely bound by $\left\{\begin{array}{lllll}0 & 0 & 0 & 1\end{array}\right\}$ planes of the neighboring zinc crystals, which seems to indicate that during solidification of these coatings in the IR furnace, zinc grains nucleated in a basal configuration at the free liquid-gas surface of these small holes.

A very similar phenomenon was observed by White [10] in a sessile drop solidification experiment. This author reported that even though the drop was cooled through the chilled substrate on which it was deposited, grains also nucleated on the opposite side at the free liquid surface in contact with the atmosphere. Platelet-shaped crystals formed there, all with their $\left\{\begin{array}{llll}0 & 0 & 0 & 1\end{array}\right\}$ plane parallel to the free surface of the drop. This is exactly the same situation as roses in galvanization, but with a concave instead of a convex liquid-air interface.

Both phenomena can be rationalized in terms of the same nucleation mechanism. White's experiment shows that interface energy effects stabilize the solid at the free surface. There is no other reason for forming solid there, since the chill is located on the opposite side of the drop and the undercooling is maximal at the interface with the chill and minimal at the free surface of the drop. Thus, $\Delta \gamma$ must be positive. In this case, the mathematical developments in the previous section predict that nucleation is favored when the $\left\{\begin{array}{llll}0 & 0 & 0 & 1\end{array}\right\}$ plane is parallel to the liquid surface, thus explaining the preferential basal orientation of the nuclei.

\subsubsection{Oxide cracks}

Another phenomenon illustrates the influence of anisotropy on zinc nucleation. Several samples, which were hotdipped in a Rhesca ${ }^{\circledR}$ galvanizing simulator, exhibit shiny stripes on parts of their surface. These macroscopically look like an array of cracks, an impression which is confirmed when they are observed in more detail by optical microscopy (Fig. 5): the upper and lower boundaries of the smooth area perfectly match each other, as the lips of a tear. Accordingly, the stripes can be interpreted as breakage of the oxide skin due to liquid flow along the vertical sample during removal from the galvanizing bath in the Rhesca experiment.

Crystal orientation measurements using electron backscattered diffraction (EBSD) reveal that the shiny bands have their $\left\{\begin{array}{llll}0 & 0 & 0 & 1\end{array}\right\}$ crystal plane parallel to the surface, while the remaining coating has a random orientation (Fig. 6). Additionally, the morphology of the surface is different in the cracks and in the coating below the remaining oxide skin: in the former, it is made of $\left\{\begin{array}{llll}0 & 0 & 0 & 1\end{array}\right\}$ facets, but 

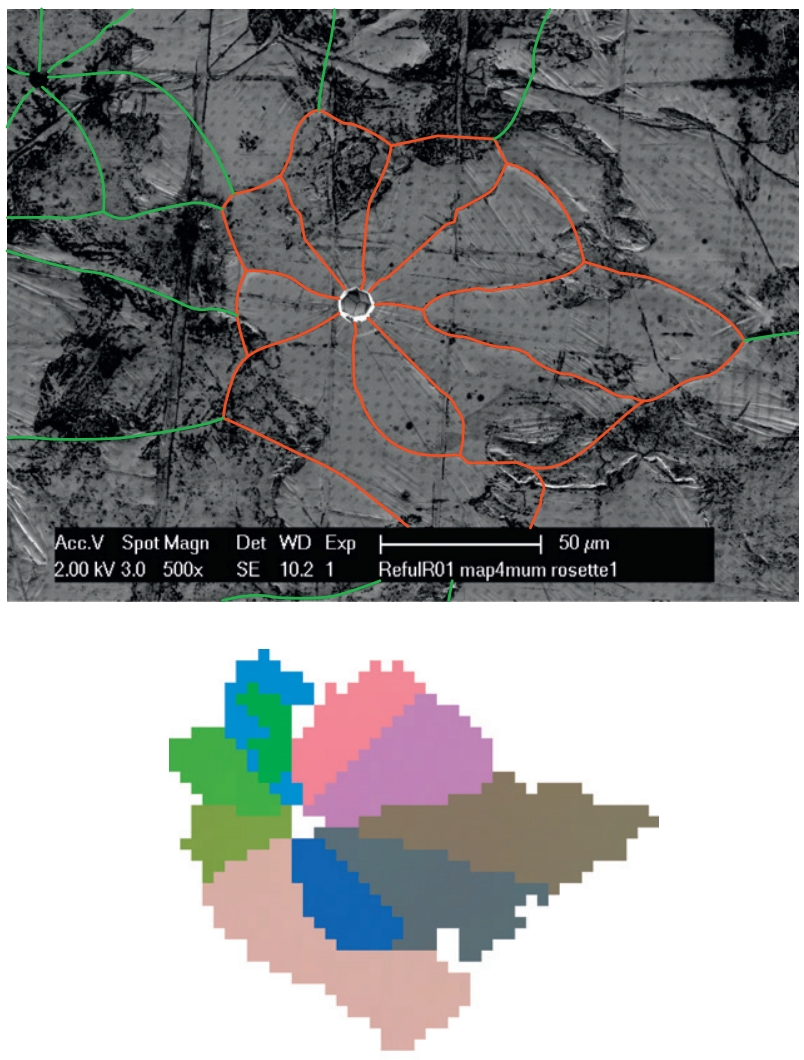

$100 \mu \mathrm{m}$

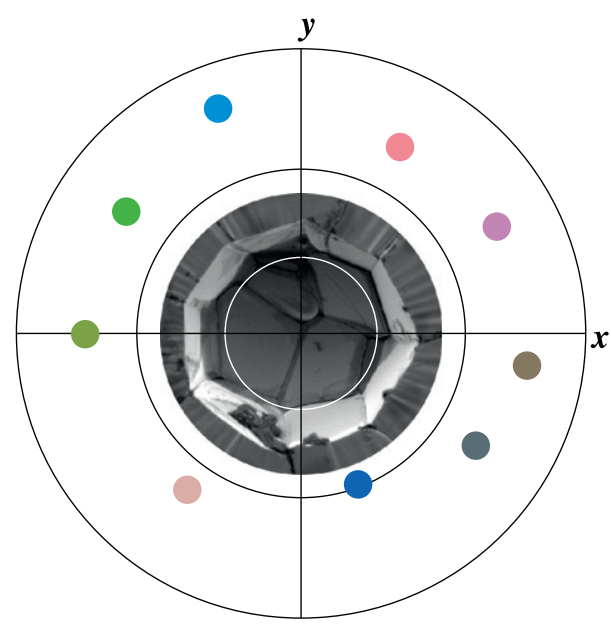

Fig. 4. Grains arranged in a rose pattern in a hot-dip galvanized coating remelted in an IR image furnace. Top: scanning electron micrograph of the coating surface, with delineated grain boundaries. Center: reconstructed electron backscattered diffraction orientation map of the grains forming the rosette pattern. The orientations are given by dots of the corresponding color in the pole figure below. Bottom: enlarged micrograph of the central cavity, with superimposed $\left\langle\begin{array}{lllll}0 & 0 & 0 & 1\end{array}\right\rangle$ pole figure of the zinc grains. Note that all poles are perpendicular to the facets of the hole, therefore indicating that the latter are $\left\{\begin{array}{llll}0 & 0 & 0 & 1\end{array}\right\}$ planes.

in the latter, it is clearly dendritic. This transition is also observed within single grains, which are at the boundary between a crack and the regular (oxidized) coating. Inter-

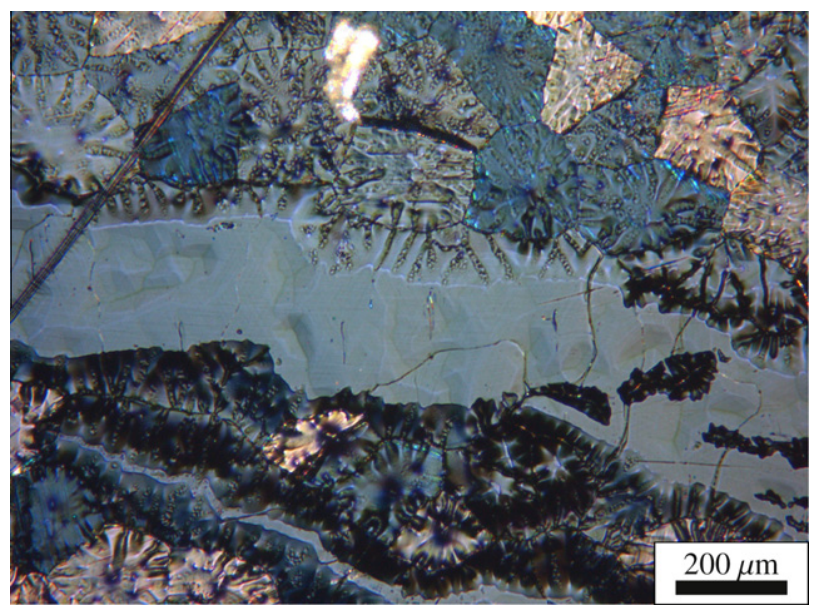

Fig. 5. Optical micrograph of a galvanized coating with broken oxide layer. Inside the tear, the surface is made of $\left\{\begin{array}{lllll}0 & 0 & 0 & 1\end{array}\right\}$ facets of zinc.

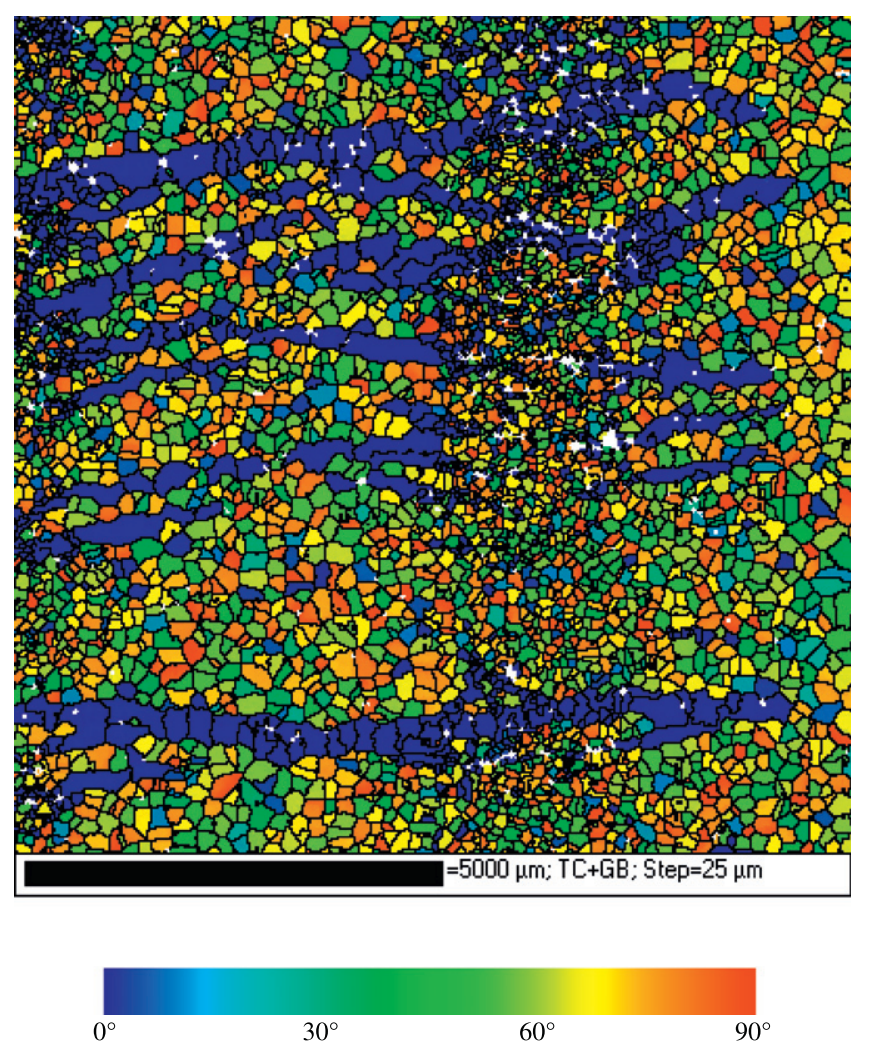

Fig. 6. Electron backscattered diffraction map of a galvanized coating with a broken oxide layer. The color gives the angle between the $\vec{c}$-axis and the normal to the sheet. The orientation of the grains inside the tears is perfectly basal $\left(0^{\circ}\right)$, while it is random in the surrounding regions.

estingly, the dendrites of such grains all point from the lips of the tear into the regular coating, indicating that the grains started within the crack and then grew under the remaining oxide layer. These observations show that nucleation of basal grains on a free surface is favored with respect to the other orientations, as predicted by the theoretical model of anisotropic heterogeneous nucleation. 


\section{Conclusion}

The extended nucleation model presented in Section 2 offers a well-defined mathematical framework that includes the effects of anisotropy on nucleation. While it demonstrated the influence of orientation, it also generalizes some of the relations which were established in the isotropic model:

- the critical nucleus radius is given by the same expression for isotropic and anisotropic nuclei;

- the free energies of heterogeneous and homogeneous nuclei of the same size are proportional, and their ratio is a function of the wetting condition $\Delta \gamma / \gamma_{s l}^{\circ}$ (and the orientation $\vec{n}_{f s}$ for anisotropic nuclei) only;

- the free energy of a nucleus is proportional to its volume.

The application of this model to zinc, besides providing an example for its use, also sheds new light into the question of galvanized coating textures. While it is commonly known among steelmakers that such coatings have a dominantly basal texture, this phenomenon has been incompletely explained until now. The examples given at the end of this paper show that the proposed model is actually at work in this system, at least locally. Due to missing data on the interfacial energies at the top surface of coatings, it cannot be determined if this mechanism applies on the whole coating surface and if it is responsible for the basal texture. However, it represents a promising new input for this research.

\section{Acknowledgements}

Grain orientation measurements were performed at the Centre interdisciplinaire de microscopie électronique of
École Polytechnique Fédérale de Lausanne, Switzerland. The authors would like to thank ArcelorMittal, and in particular M. Bobadilla, for their financial support and scientific interest to this work.

\section{References}

[1] Turnbull D. Kinetics of heterogeneous nucleation. J Chem Phys 1950;18(2):198-203. doi:10.1063/1.1747588.

[2] Greer AL, Bunn AM, Tronche A, Evans PV, Bristow DJ. Modelling of inoculation of metallic melts: application to grain refinement of aluminium by Al-Ti-B. Acta Mater 2000;48:2823-35.

[3] Quested TE, Greer AL. Athermal heterogeneous nucleation of solidification. Acta Mater 2005;53:2683-92.

[4] Taylor JE, Cahn JW. Theory of orientation textures due to surfaceenergy anisotropies. J Electron Mater 1988;17(5):443-5.

[5] Hoffman DW, Cahn JW. A vector thermodynamics for anisotropic surfaces. 1. Fundamentals and application to plane surface junctions. Surf Sci 1972;31:368-88. doi:10.1016/0039-6028(72)90268-3.

[6] Cahn JW, Hoffman DW. A vector thermodynamics for anisotropic surfaces. 2. Curved and faceted surfaces. Acta Metall 1974;22(10): 1205-14. doi:10.1016/0001-6160(74)90134-5.

[7] Miller WA, Chadwick GA. The equilibrium shapes of small liquid droplets in solid-liquid phase mixtures: metallic h.c.p. and metalloid systems. Proc Roy Soc Lond Ser A 1969;312(1509):257-76. <http:// www.jstor.org/stable/2416221>.

[8] Keşlioğlu K, Maraşlı N. Experimental determination of solid-liquid interfacial energy for $\mathrm{Zn}$ solid solution in equilibrium with the $\mathrm{Zn}-\mathrm{Al}$ eutectic liquid. Metall Mater Trans A 2004;35(12):3665-72. doi:10.1007/s11661-004-0272-8.

[9] Mariaux A. Texture formation in hot-dip galvanized coatings: nucleation and growth of anisotropic grains in a confined geometry. Ph.D. thesis 4646. Lausanne (Switzerland): École polytechnique fédérale de Lausanne; 2010.

[10] White DWG. Surface tension and the solidification behaviour of zinc and cadmium. J Inst Met 1971;99:287-9. 